\section{Time to put periodontal disease on the list of chronic inflammatory diseases contributing to premature atherosclerosis}

Legge and Hanly are to be commended for an excellent review on the management of premature atherosclerosis in patients with chronic inflammatory disease. ${ }^{1}$ Perhaps the review would be more complete if it included a substantive discussion on the potential for an individual's cumulative burden of inflammation to be amplified by periodontal disease.

The most common form of periodontal disease is chronic inflammatory periodontal disease. A multifactorial disease, chronic inflammatory periodontal disease begins with a microbial infection on tooth surfaces, followed by a host-mediated destruction of adjacent tissues. Cells that mediate inflammation generate cytokines, eicosanoids and matrix metalloproteinases that cause clinically significant connective tissue and bone destruction. ${ }^{2}$

Cytokine-induced destruction of collagen leads to not only the loss of the investing bone, but also damage to the connective tissue supporting the lining epithelium approximating the tooth. In this typical periodontal lesion, the integrity of the lining epithelium is compromised, allowing bacteria, bacterial byproducts and the cytokines released in response to the initiating bacteria to penetrate regularly through the ulcerated epithelium into the bloodstream. ${ }^{3}$

The consequences of biofilm accumulation in a particular individual are influenced by various well-established risk factors for chronic inflammatory periodontal disease, which are also common to other systemic diseases, including tobacco use, obesity, nutrition, exercise, hypertension, dyslipidemia, psychosocial stress, sleep disorders, excessive alcohol consumption and changes in bone metabolism. ${ }^{4}$ Not surprisingly, emerging data continue to indicate that periodontal diseases may also be associated with a wide array of systemic diseases and conditions, including cardiovascular disease. ${ }^{5}$

Establishing causality between inflammation of oral origin and any of the associated inflammation-driven chronic diseases will continue to be challenging. To establish proof, we will need to quantify the specific level of inflammation of oral origin that contributes to the systemic inflammatory burden. Although efforts to do so are underway, ${ }^{6}$ there is still work to be done. Long-term intervention studies assessing the effect that ongoing dental therapy (aimed at helping patients achieve and maintain a functioning dentition with minimal inflammation over their life span) has on associated systemic diseases will be necessary before causality can be determined.

Nevertheless, there is now undeniable evidence that the local inflammatory response to periodontal biofilm spills into the circulatory system contributes to the level of systemic inflammation. ${ }^{3,7}$ There is also evidence that periodontal intervention can result in a reduction in systemic markers of inflammation. ${ }^{8}$ Even in the absence of hard data establishing a causal relation between periodontal disease and systemic disease, evidence certainly suggests that periodontal disease is a contributing factor to the associated systemic diseases. ${ }^{9}$

It seems prudent to add periodontal disease to Legge and Hanly's discussion concerning which factors contribute to the excessive cardiovascular disease burden they noted in patient populations. More importantly, co-managing the care of patients with risk factors for common systemic diseases like periodontal disease and cardiovascular disease may be an important part of managing both diseases. ${ }^{4}$

\section{Timothy G. Donley DDS MSD}

Periodontist, Bowling Green, Ky.;

member of the Cardiovascular Research

Foundation, New York, NY

Cite as: CMAJ 2019 January 14;191:E52. doi: $10.1503 / \mathrm{cmaj} .71062$

\section{References}

1. Legge A, Hanly JG. Managing premature atherosclerosis in patients with chronic inflammatory disease. CMAJ 2018;190:E430-9.

2. Kornman KS, Page RC, Tonetti MS. The host response to the microbial challenge in periodontitis: assembling the players. Periodontol 2000 1997; 14:33-53.

3. Tomás I, Diz P, Tobias A, et al. Periodontal health status and bacteraemia from daily oral activities: systematic review/meta-analysis. J Clin Periodontol 2012;39:213-28.

4. Friedewald VE, Kornman KS, Beck JD, et al.; American Journal of Cardiology; Journal of Periodontology. The American Journal of Cardiology and Journal of Periodontology editors' consensus: periodontitis and atherosclerotic cardiovascular disease. Am J Cardio 2009;104:59-68.

5. Nagpal R, Yamashiro Y, Izumi Y. The two-way association of periodontal infection with systemic disorders: an overview. Mediators Inflamm 2015;2015: 793898. doi: 10.1155/2015/793898.

6. Temelli B, et al. Circulation levels of acute phase proteins pentraxin 3 and serum amyloid $A$ in atherosclerosis have correlations with periodontal inflamed surface area. J Appl Oral Sci 2018;26: e20170322.

7. Cardoso EM, Rei C, Manzanares-Céspede MC. Chronic periodontitis, inflammatory cytokines, and interrelationship with other chronic diseases. Postgrad Med 2018;130:98-104.

8. Önder C, Kurgan Ş, Altıngöz SM, et al. Impact of non-surgical periodontal therapy on saliva and serum levels of markers of oxidative stress. Clin Oral Investig 2017;21:1961-9.

9. Bale B, Doneen A. High-risk periodontal pathogens contribute to the pathogenesis of atherosclerosis. Postgrad Med 2018;149:401-3.

Competing interests: None declared. 